\title{
Software toolkit for evaluating infrared imaging seeker
}

\author{
Marianne A. C. Degache \\ ${ }^{a}$ TNO, P.O. Box 96864, 2509 JG The Hague, The Netherlands
}

\begin{abstract}
Modern infrared imaging seekers can nowadays deal with higher resolution and less noisy sensor images. The testing of new image processing or tracking algorithms requires a fitted set of relevant sensor images. When no actual recordings are available, when experimental benches are not adapted or at an early stage of development, one can require a simulation tool to generate synthetic infrared sensor images.

This paper presents the first version of ISISserver (Infrared Sensor Image Simulation Server) a software library developed at TNO and used for infrared (IR) imaging seeker applications. Based on the EOSTAR Pro (Electro-Optical Signal Transmission And Ranging) model suite, the set of functions offered by this toolkit allows analysis of synthetic sensor images generated for various synthetic environments and targets. Typical targets from a database can be used as well as externally user designed 3D targets. Simulation results using ISISserver toolkit with test data (not realistic or physical data) will be shown.
\end{abstract}

Keywords: Infrared imaging seeker, Software toolkit, Infrared scene modeling

\section{INTRODUCTION}

Modern infrared seekers benefits from advanced imaging capabilities (with high resolution and low noise level) and may include post processing allowing for better detection ranges, improved tracking and optimized final outcome (for example, hits - damage or kill). The performance of their embedded image processing or tracking algorithms can be characterized by three means:

1. field tests

2. laboratory tests

3. models

Due to the costs and complexity, field tests are organized at the latest possible stage of the development cycle. Laboratory tests require adapted experimental benches to address the relevant issues that can challenge algorithms and processing. Because of the limitations in the optical set ups simple cases must be considered and most of the sensitivity studies reflecting adverse conditions cannot be conducted during such tests. The main advantages of models are the costs (provided background knowledge and existing models can be utilized) and the flexibility to consider multiple and different scenarios. Another feature of the modeling is the possibility to repeat simulations and to extend analysis of the results (including intermediate results). A first example is a hybrid model including actual recordings that can be used as input. Another example is a generator of infrared images combined with standard or new image processing algorithms. The variability of these synthetic images can be guaranteed by the model itself while the modeling environment can integrate upgrades focusing on specific concerns. As a result, the use of a simulation tool for the testing of IR seeker data processing algorithms can be extremely valuable.

In the last few decades, the former TNO branch "Defence, Security and Safety" had a need for different models for IR sensor, IR seeker, missile fly out or IR scene. For that reason, elements of software have been acquired or developed in house to meet these needs. Gathering such elements is the initial step towards the creation of a toolkit addressing purposes such as Seeker performance studies, Hit-Point analysis or Kill assessment. The next step is the identification of individual components so that the interfaces can be established to enforce modularity. Finally, the toolkit can be defined and implemented for a use in an application. For all practical purposes the preferred software architecture is a clientserver architecture.

Infrared Imaging Systems: Design, Analysis, Modeling, and Testing XXII, edited by Gerald C. Holst, Keith A. Krapels, Proc. of SPIE Vol. 8014, 80140E · (c) 2011 SPIE · CCC code: 0277-786X/11/\$18 · doi: 10.1117/12.884487 


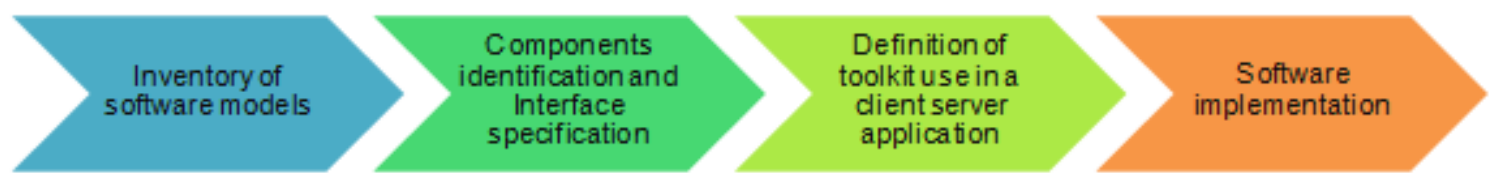

Figure 1. Development process of a software toolkit

The following sections will introduce the software elements that have been used for the development of a toolkit to evaluate infrared imaging seeker. A test case is then given for an exo-atmospheric interceptor scenario.

\section{PRE-EXISTING SOFTWARE}

On the one hand, TNO has extensive experience on the development of models to assess missile and countermeasure performance for both RF and IR. For a couple of projects, IR imaging seeker models have been implemented and tested in house either on real data (SURFER project, see Broek et al. $(2000)^{[2]}$ ) or simulated data from a very modest scene generator (see the countermeasure methodology presented by Schoemaker et al. (2010) $)^{[8]}$.

On the other hand, an electro-optical model suite: EOSTAR (Electro-Optical System Transmission And Ranging) is being developed since 2001 at TNO (see Kunz et al. 2004) ${ }^{[5]}$. The models in EOSTAR have been primarily used to assess the impact of the environment on military EO sensors in for the Navy's operations. In its current status, the suite covers the whole chain of observation and includes detailed atmospheric effects like molecular and aerosol transmission losses, refraction and turbulence but also thermal camera models as well as a physically based IR scenery generation (see Degache et al. 2010) $)^{[3]}$.

EOSTAR modular design can address various scenario types ranging from a calculation of propagation losses on a given path, a prediction of the operational signature of a marine platform up to the detection range of a specific threat in a region of interest. For the purpose of this work, the following scenario (see Figure 2) is considered: a simple IR scene with a single target against a background is observed by a sensor and an automated detection scheme is applied to this observation.

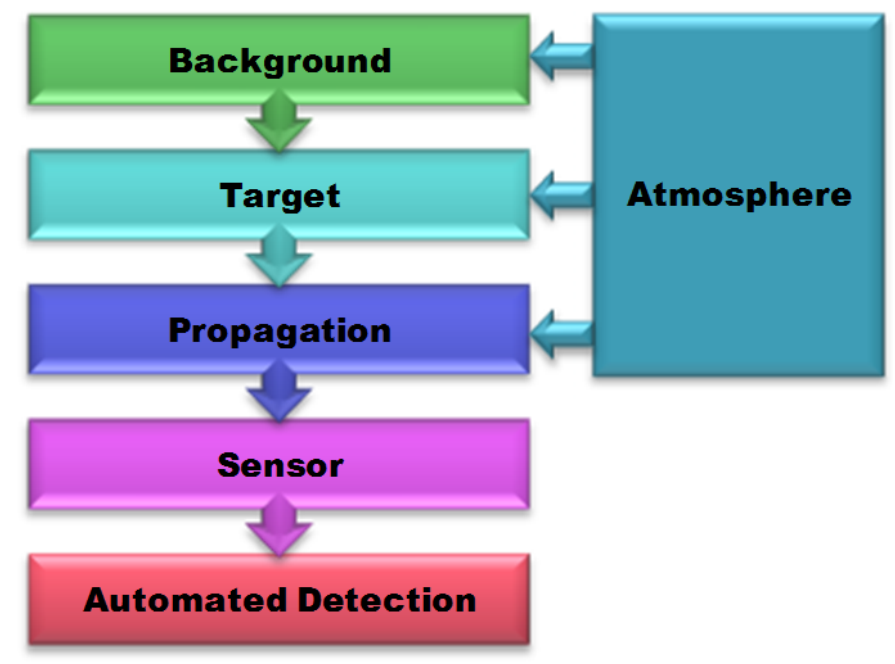

Figure 2. EOSTAR Pro block schema for an IR seeker detection scenario 
The main output of this EOSTAR Pro scenario is a representative (synthetic) sensor image and the corresponding detection probability. These results can be displayed to the user thanks to a graphical user interface, part of the standard EOSTAR Pro package. Examples are given in Figure 3 in the case of maritime scenario.
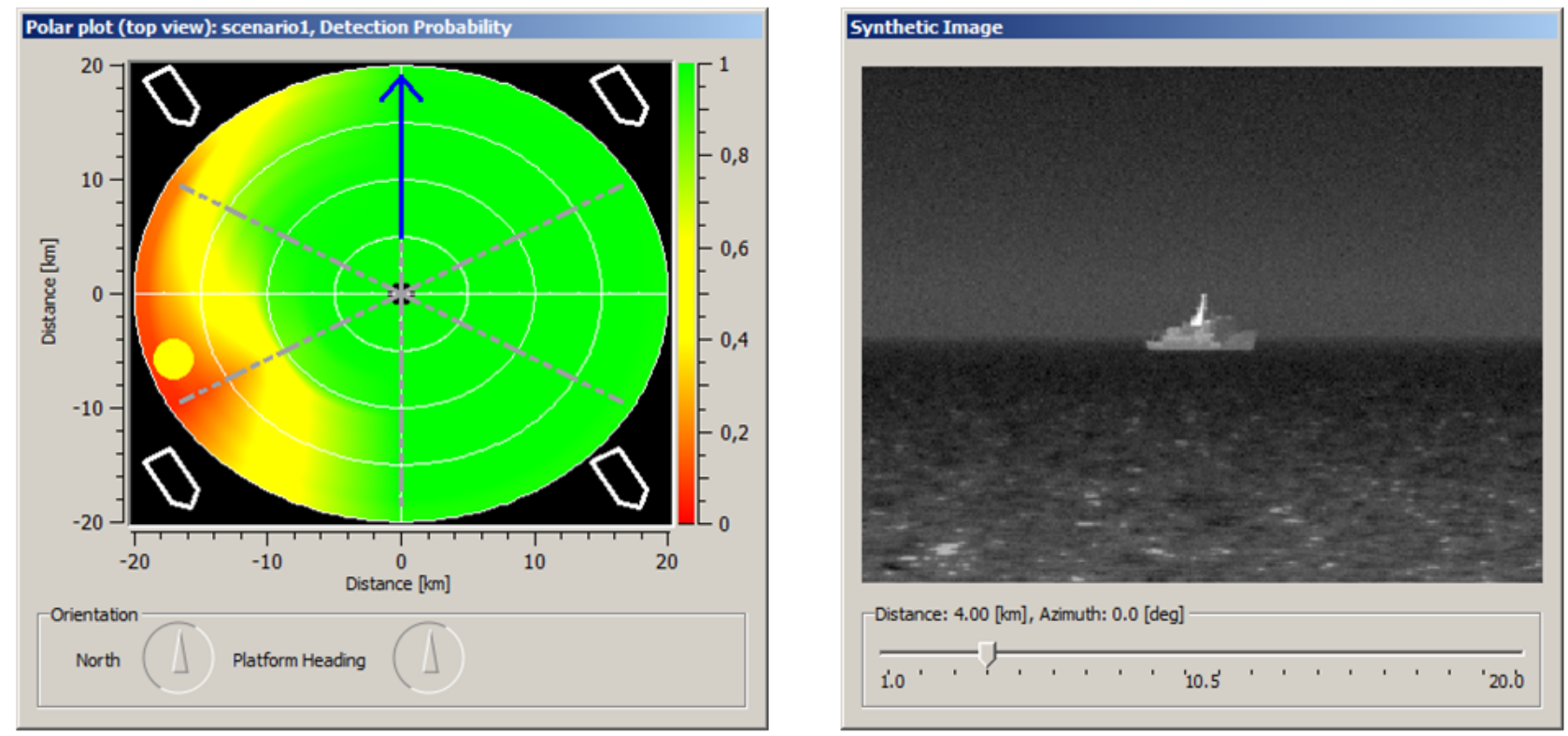

Figure 3. EOSTAR Pro outputs: left part shows the detection probability of the "Quest" target by a mid wave infrared sensor on a $20 \mathrm{~km}$ range radius; right part shows the predicted sensor image on a given position (range, azimuth).

The direct exploitations of such outputs have been proven useful for external applications such as a target acquisition model in the EO-VISTA software framework (see Bijl et al., 2008) ${ }^{[1]}$ or a complete missile fly-out simulator with the TNO Electronic Warfare Model EWM (see the hit point analysis by Schoemaker et al., 2010) ${ }^{[8]}$. However this type of coupling was limiting since the applications were forced to consider open loop simulations and prevented more advanced studies on dynamic scenarios. Considering the considerable acquired knowledge on this topic and the definition of new requirements for future simulators, a decision was taken to upgrade EOSTAR in order to improve software reusability and address a larger variety of applications. The first development of a software toolkit based on EOSTAR Pro and to be used in closed loop scenarios started in 2010.

\section{SOFTWARE TOOLKIT DEVELOPMENT}

The block schema referred in Figure 2 shows that the end-to-end approach of EOSTAR does cover the whole detection chain. However it was developed for surface-to-surface scenarios in a maritime environment. For EOSTAR to handle different types of scenarios (air-to-air, air-to-surface or surface-to-air) - possibly in different environments - major upgrades are necessary so as to include new and different physics. The first requirements for such upgrades are a good modularity and the achievement of the user specified performance. This can be fulfilled by following the steps towards the development of a software toolkit (step 2 to 4 in Figure 1).

\subsection{Components identification and interface specification}

The components needed for a client application using EOSTAR Pro externally can be easily identified as the different blocks in Figure 2. However the application must specify the system to be modeled by EOSTAR Pro not only by its required components (all or some of them) but also by the selection of models to be used internally by EOSTAR Pro for each component. All of it is covered by a scenario specification in EOSTAR that is user-specific. 
Many of these external applications are exploiting the output synthetic image (Figure 3) of a specified EOSTAR Pro scenario. In addition to these physically modeled images, specific intermediary or post processed outputs can be required. A specific interface for each of these data sets needs to be specified.

\subsection{Toolkit use in a client-server application}

The toolkit is defined with the interface required by the client application. Its goal is to define the relevant EOSTAR Pro scenario, call EOSTAR Pro functions/models and access the corresponding input and output data. The main current interest for the toolkit is to provide a synthetic image so it was named ISISserver (the model generating the final sensor image in EOSTAR is called ISIS, standing for Infrared Sensor Image Simulation).

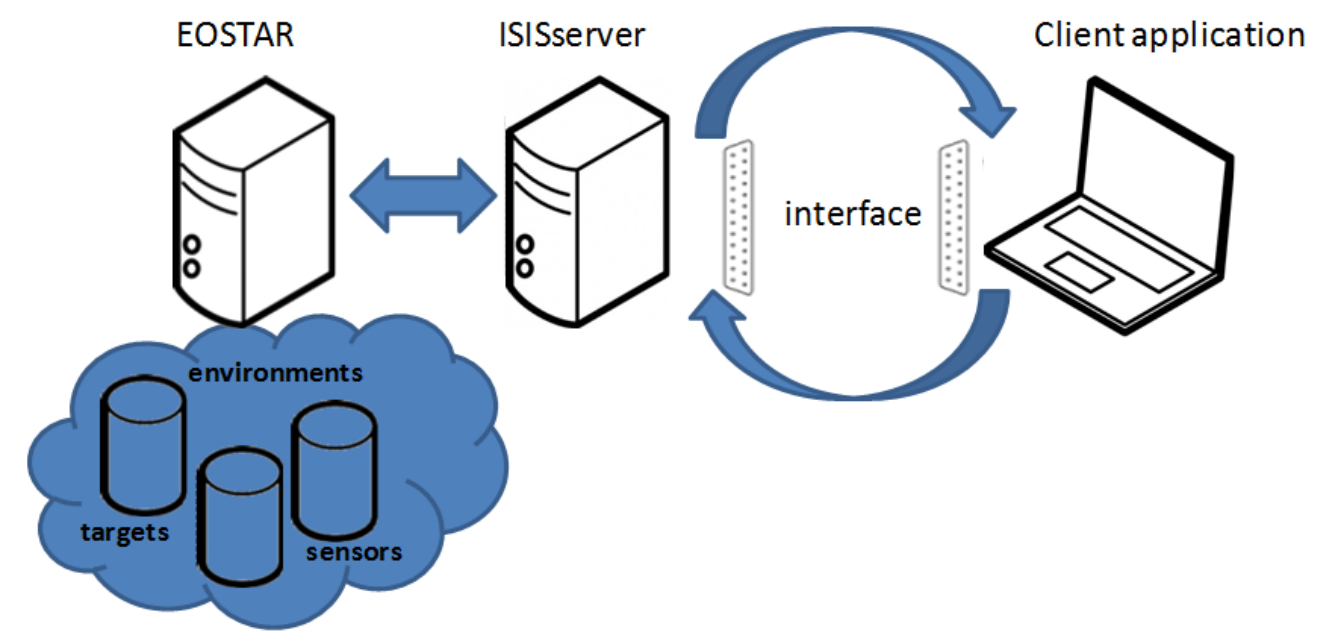

Figure 4. ISISserver toolkit communication

\subsection{Software Implementation}

ISISserver is written in $\mathrm{C}++$ and, as shown is Figure 3, is directly linked (static link) to EOSTAR Pro kernel and libraries. This allows sharing internally the different data stores and the object classes used by the models. It also allows an easy access to the databases used by EOSTAR (especially target and sensor databases).

ISISserver is compiled and dynamically linked as a library (DLL). It makes use of the Microsoft standard calling convention for the client application's requests and data exchange.

\section{SIMULATIONS}

The first version of ISISserver has been tested on simple scenarios for a missile interception. No real values for the parameters have been taken since the goal is to illustrate the principle and not interpret simulations of a real system.

\subsection{Missile interception test scenarios}

1. Scenario components

A missile interception test scenario has been defined with the following components:

- One target representing a missile (a generic CAD model with an arbitrary - not realistic - thermal signature has been defined for the testing)

- One imaging infrared sensor (mid wave (MWIR) or long wave infrared (LWIR) wavebands)

- Additional sensor processing providing the aiming point for a missile guidance system 
In this scenario, the atmosphere is neglected (an assumption is made here that the interception occurs in the middle course phase when the missile is in the exosphere). The background in the sensor field of view is considered as being only constituted by the cold sky. For a scenario, a sequence of images is considered to simulate dynamics for the target or the sensor.

The following scenarios are using the same components (same generic missile target, same generic LWIR sensor) but for various sensor-target geometries:

2. "Perfect aiming" test scenario

The "perfect aiming" test scenario considers a generic IR seeker getting closer to a generic missile target. The initial position of the target is in the center of the field of view and the sensor is aiming perfectly towards the center of the target.

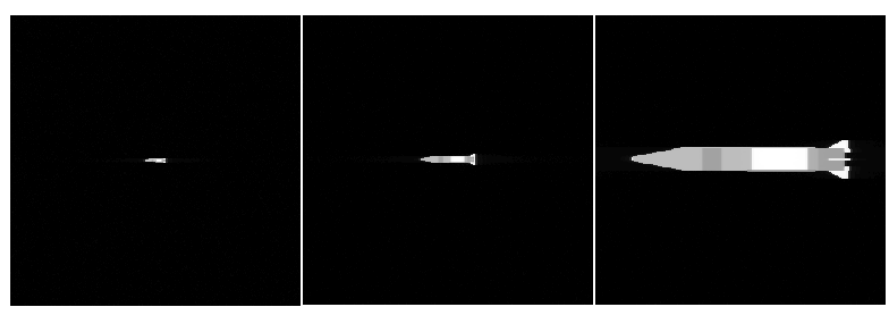

Figure 5. "Perfect aiming" test scenario, sample images

3. "Oscillating target height" test scenario

The "oscillating target height" test scenario considers a generic IR seeker aiming to a generic missile target. The sensor is aiming perfectly towards the center of the target in one direction (horizontal) and is oscillating on the other direction (vertical). The range remains constant.

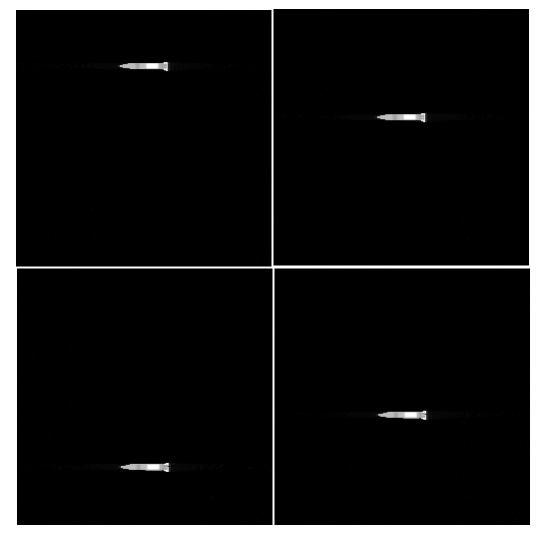

Figure 6. "Oscillating target height" test scenario, sample images

4. "Yaw-rotation" test scenario

The "yaw-rotation" test scenario considers a generic IR seeker aiming to a generic missile target with various yaw angles. The sensor is aiming perfectly towards the center of the target. The range remains constant.

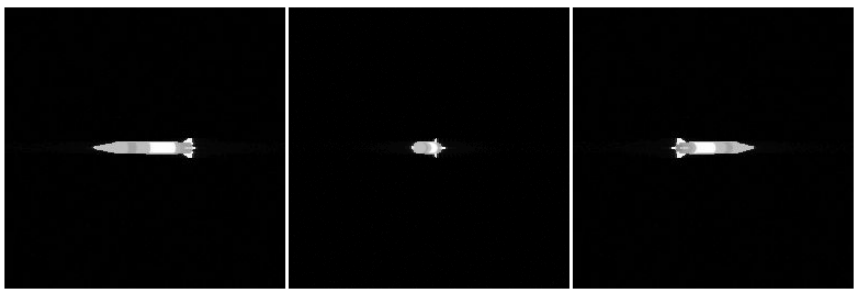

Figure 7. "Yaw-rotation" test scenario, sample images 


\section{5. "Pitch-rotation" test scenario}

The "pitch-rotation" test scenario considers a generic IR seeker aiming to a generic missile target with various pitch angles. The sensor is aiming perfectly towards the center of the target. The range remains constant.

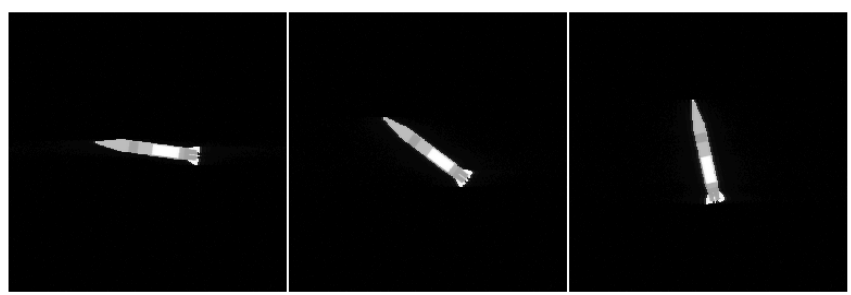

Figure 8. "Pitch-rotation" test scenario, sample images

\subsection{Infrared Homing tests}

To demonstrate the use of the toolkit for evaluating imaging seekers, three simple image processing models analyzing the synthetic sensor images have been developed. Each model computes and provides an aiming point for a missile guidance system:

- Model A: the aiming point is selected as the 'hottest' pixel of the sensor image.

- Model B: the aiming point is selected as the center of the detected target (center of the segmented target image after thresholding)

- Model C: the aiming point is selected as the barycenter of the sensor image excluding outliers.

\subsection{Results comparison}

- "Perfect aiming" test scenario
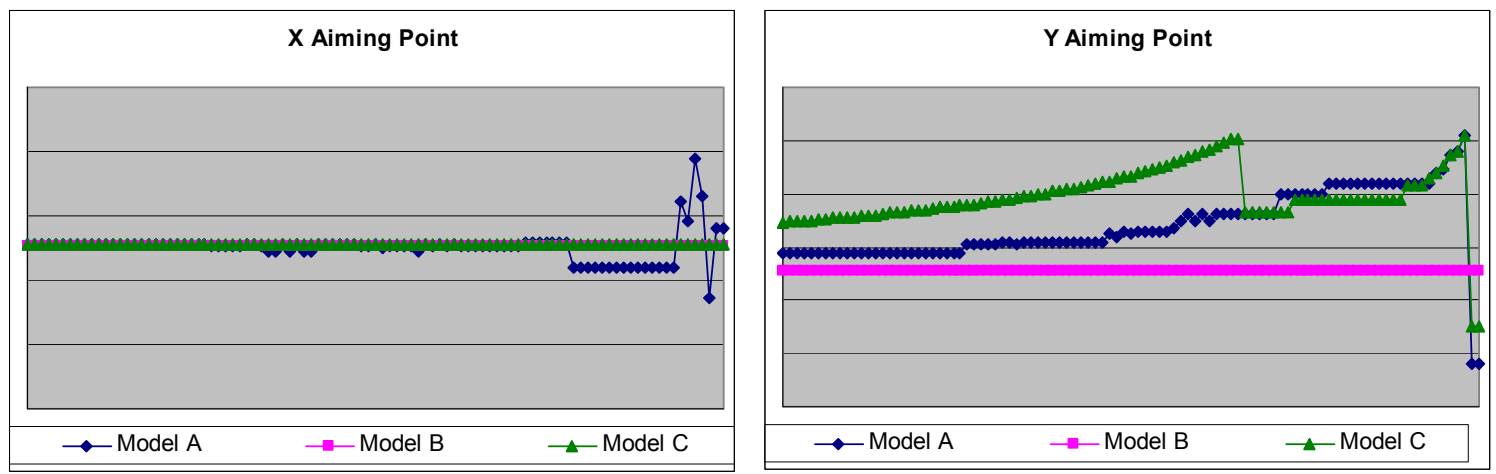

Figure 9. "Perfect aiming" test scenario, models results

As we can see in Figure 9, the horizontal coordinate of the aiming point ("X", corresponding to a position in the image rows) for the "perfect aiming" test scenario remains nearly constant for all models. The vertical coordinate of the aiming point ("Y" ", corresponding to a position in the image columns) is similar for all models when the target apparent size is small (a few pixels in the sensor image) and diverges for Model A and Model C when the target apparent size is larger. This is explained by the thermal contrast observed in the selected missile signature as seen in Figure 10. Indeed the hottest point of the target does not correspond to the center of the geometrical model so there is an offset between Model A and Model B that depends on the range. Model C aiming point differs from Model A because it considers not only the hottest spot but the whole thermal regions of the target image. Finally by the end of the scenario, there is a shift caused by the fact that only a part of the target is visible in the sensor image so the contrast for the hot spots and regions is altered. 

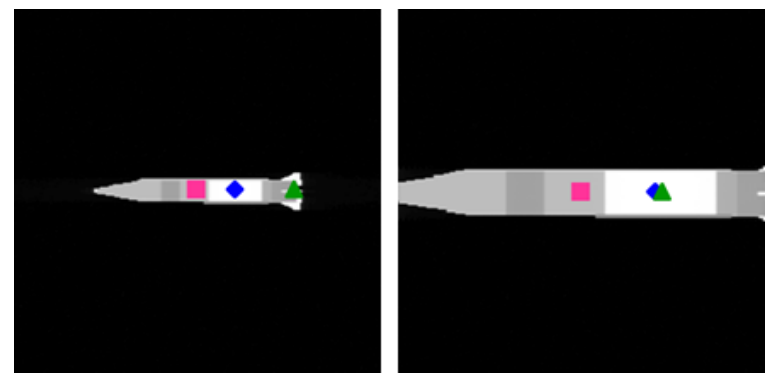

Figure 10. "Perfect aiming" test scenario, aiming point for two individual image frames. As stated before, the images do not reflect any realistic missile infrared signature. The blue diamond corresponds to Model A, the pink square to Model B and the green triangle by Model C.

- "Oscillating target height" test scenario
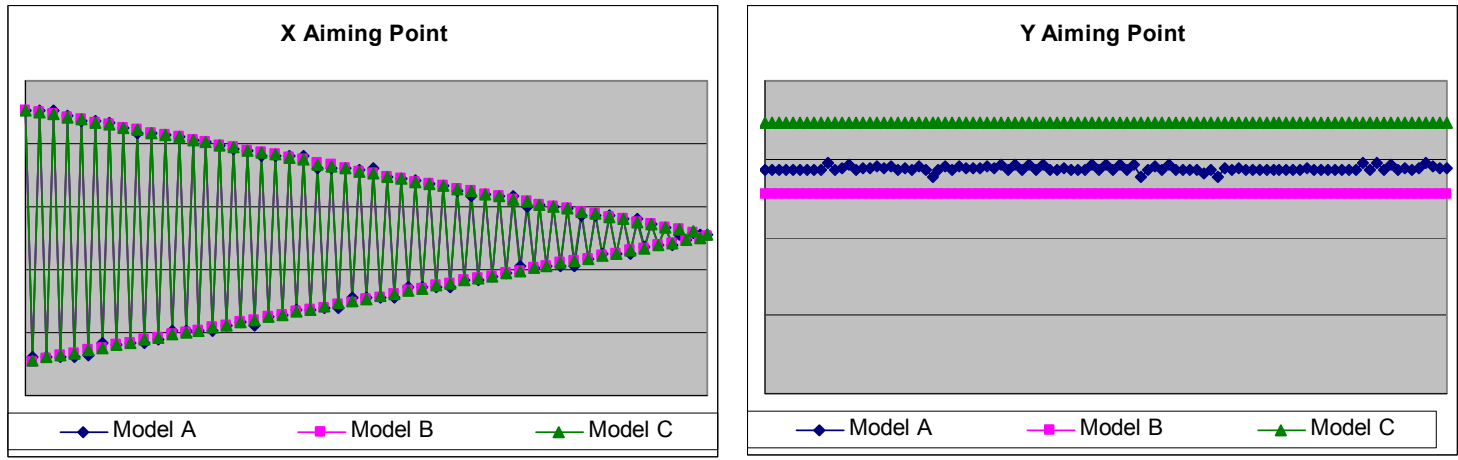

Figure 11. "Oscillating target height" test scenario, models results

As we can see in Figure 11, there is no significant difference of behavior for the three models for "oscillating target height" test scenario. The aiming point has only an offset in the "Y" direction.

- "Yaw-rotation" test scenario
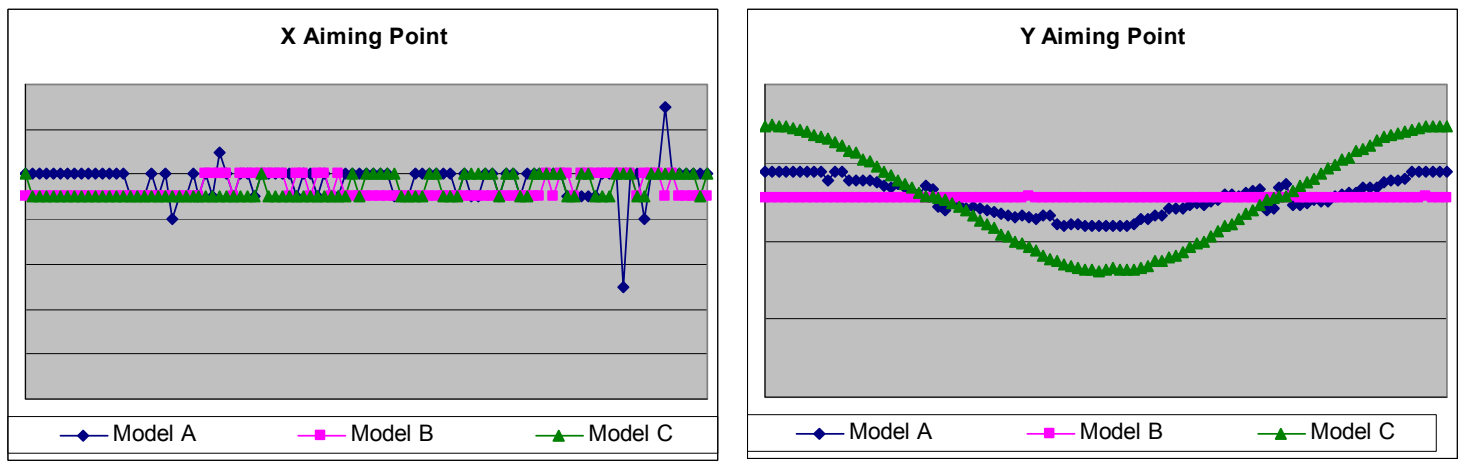

Figure 12. "Yaw-rotation" test scenario, models results

For the "Yaw-rotation" test scenario, the vertical coordinate of the aiming point ("Y") is constant for the model B. Indeed the target is rotating on itself so the center of the target image is not modified despite the change of aspect angle. For model $\mathrm{A}$ and $\mathrm{C}$ however, the visibility and the location of the hot spots in the target image is modified so the vertical coordinates of the aiming point is modified accordingly.

- "Pitch-rotation" test scenario 

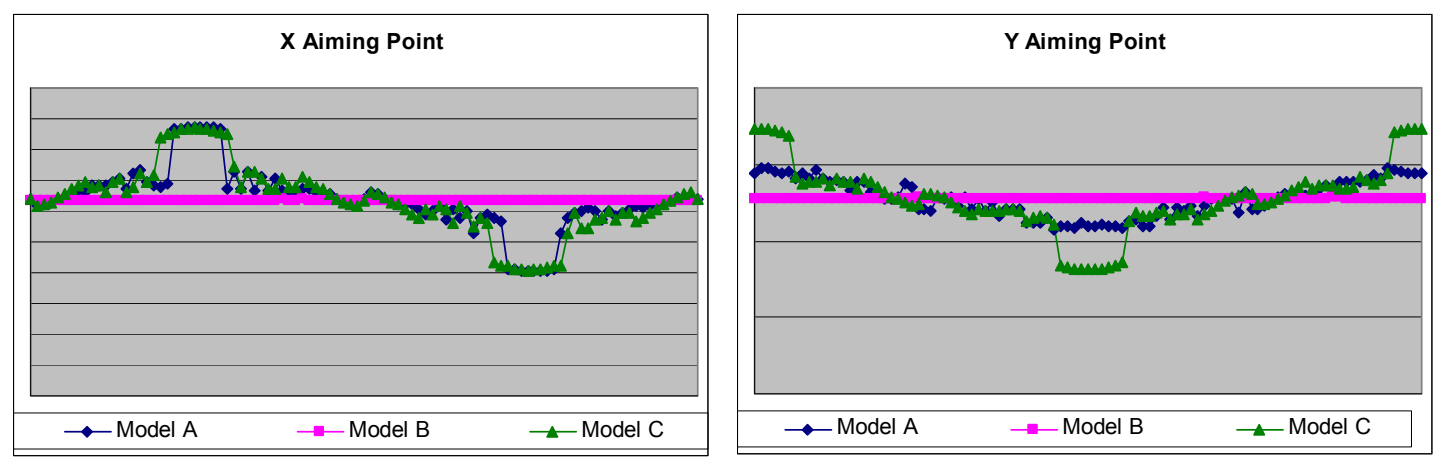

Figure 13. "Pitch-rotation" test scenario, models results

For the "Pitch rotation" test scenario, a pattern can be observed in the "X" and the "Y" directions for model A and model C. This is caused by the strong variation of the apparent dimension of the target in that direction (for example, for a rotation of 90 degrees, the dimension of the missile in the $\mathrm{X}$ direction will correspond to the whole length of the missile and in the $\mathrm{Y}$ direction the dimension will correspond to the missile diameter.

\subsection{Performance}

The primary goal of this toolkit is to provide the required information to an external application within a reasonable amount of time. When extensive simulations are needed, the expected performance of such toolkit is 'near real time'. The demonstrator build for this project has been run on a standard laptop with a $1.6 \mathrm{GHz}$ Intel Core 2 Duo processor with an Intel integrated graphic card. Despite the machine limited performance, ISISserver was able to deliver 15 sensor images (and the aiming point calculation) per minute. These results are promising for a demonstrator that has not been optimized.

\section{CONCLUSION AND FUTURE WORK}

This paper has introduced the concept of a toolkit based on the TNO electro-optical sensor performance model suite EOSTAR Pro. A demonstrator "ISISserver" has been build and released for a client-server application focusing on an exo-atmospheric interceptor scenario.

ISISserver has demonstrated that the modular approach of a simulation toolkit can help the developer to easily derive a new scenario and a new application. By combining existing software elements in a dedicated server, ISISsever was able to provide a quick solution for specific simulation needs.

A few test scenarios have been discussed in this paper. As a demonstration case, ISISserver has also been recently connected to a missile guidance model ${ }^{9}$. The first version of this toolset is promising as much for its simulation possibilities as its current reasonable speed performance.

\section{ACKNOWLEDGEMENTS}

The author wishes to thank Wouter Halswijk and Marco van der Lans for their support and the stimulating discussions on missile modeling. The author is also grateful for Ric Schleijpen inputs on seeker models and the overall exoatmospheric interceptor scenario definition.

Finally we thank the EOSTAR team for providing the modules from which ISISserver has been built. 


\section{REFERENCES}

[1] Bijl, P., Hogervorst M. A. and Vos, W. K., "Modular Target Acquisition model \& visualization tool," Proc. SPIE 6941, (2008).

[2] Van Den Broek, S. P., Bakker, E. J., De Lange, D. and Theil, A. "Detection and classification of infrared decoys and small targets in a sea background", Proc. SPIE 4029, pp70-80 (2000)

[3] Degache, M. A. C., Van Eijk, A. M. J., Tsintikidis, D. and Hammel, S. M., "EOSTAR Pro: a flexible extensive library to assess EO sensor performance" Proc. SPIE 7828-03 (2010)

[4] Halswijk, W., "Application of the high-fidelity electro-optical model suite EOSTAR to an exo-atmospheric interceptor scenario" 7th International Conference on Missile Defence "Challenges in Europe" (2011)

[5] Kunz, G. J., Degache, M. A. C., Moerman, M., Van Eijk, A. M. J., Neele, F. P., Doss-Hammel, S. M. and Tsintikidis, D., "Status and developments in EOSTAR a model to predict IR sensor performance in the marine environment", Proc. SPIE 5572, pp. 101-111 (2004).

[6] De Jong, W., Dam, F. A. M., Kunz, G. J. and Schleijpen, R.M.A., "IR seeker simulator and IR scene generation to evaluate IR decoy Effectiveness", Proc. SPIE 5614-11, (2004).

[7] Mieremet, A. L. and De Jong, W., "EOSM validation using SAPPHIRE data", TNO report TNO-DV 2008 A253, June 2008, NATO restricted (2008).

[8] Schoemaker, R. and Schleijpen, R.M.A., "Evaluation Tools for the Effectiveness of Infrared Countermeasures and Signature Reduction for Ships", Proc. SPIE 7662 paper 26 (2010). 